\title{
Plasmonic switch Based on asymmetric cavities with embedding square of gold inside the cavities
}

Majid Ghadrdan ( $\square$ ghadrdan@ece.usb.ac.ir )

University of Sistan and Baluchestan https://orcid.org/0000-0002-6135-0317

Mojtaba Shahraki

University of Sistan and Baluchestan

Mohammad Ali Mansouri-Birjandi

University of Sistan and Baluchestan

Original Research

Keywords: cavity, gold nonlinear properties, nonlinear Kerr effect, plasmonic switch

Posted Date: February 1st, 2021

DOl: https://doi.org/10.21203/rs.3.rs-180545/v1

License: (c) (i) This work is licensed under a Creative Commons Attribution 4.0 International License.

Read Full License 


\section{Abstract}

In this paper, we proposed an all-optical plasmonic switch based on metal-insulator-metal (MIM) structures. We used the intrinsic nonlinear properties of gold to implement the switch. The proposed switch consists of a bus waveguide side coupled with a pair of asymmetric vertical cavities. We obtained the transmission spectrum of the structure for low input intensities. The results showed that a sharp dip occurs at the wavelength of $860 \mathrm{~nm}$. Due to the nonlinear properties of gold and the nonlinear Kerr effects, the proposed switch has a high transmission ratio of about 0.8 and a low threshold power of $0.07 \mathrm{~mW} / \mu \mathrm{m}^{2}$. The threshold power of the structure with and without using the gold nanostructure shows a reduction of $50 \%$. The result showed that the proposed switch has the potentiality to be applied in the plasmonic integration circuits.

\section{Introduction}

All-optical switches are indispensable in the integrated optical circuits and are widely applied in all-optical networks (Sasikala and Chitra 2018). The proposed structures for implementing optical switches operate based on different principles with different characteristics and applications, among which, optical switches based on the excitation of Surface plasmon polaritons have attracted attention due to their fast response time, low power consumption, and nanometer-scale (Emadi et al. 2017; Ghadrdan and Mansouri-Birjandi 2016; Ghadrdan and Mansouri-Birjandi 2017; Nurmohammadi et al. 2018; Zhang and Yang 2019; Shahamat and Vahedi 2018). Surface plasmon polaritons (SPPs) are electromagnetic waves propagating at the interface between a metal and a dielectric material. In the metal structures, only TM polarization excites the SPPs. When light with TM polarization is applied to the metal structure, SPPs are excited (Kik and Brongersma 2007; Maier 2007). Add-drop filters, logic gates, multiplexers, and specifically switches are various types of plasmonic devices that attracted lots of scientist's attentions (Bashiri and Fasihi 2019: Fasihi 2014; Ghadrdan and Mansouri-Birjandi 2013; Mansouri-Birjandi et al. 2016; Monfared et al. 2020; Negahdari et al. 2019). Plasmonic switches are in the range of nanometres with fast response, low input power, and high transmission efficiency. One of the important and interesting approaches for the implementation of plasmonic switches is the optical nonlinear Kerr effect. Plasmonic switches are implemented based on cavities (Hocini et al. 2020; Kwon t al. 2014; Paul and Ray 2016), Mach-Zehnder interferometers (Janjan e al. 2017; Wu et al. 2015), directional couplers (Nozhat and Granpayeh 2012; Petráček 2013), and ring resonators (Ghadrdan and Mansouri-Birjandi 2018; He et al. 2015; Nozhat and Granpayeh 2014; Wu et al. 2014). In this research, we demonstrate an all-optical switch based on the plasmonic cavity. It has the potentiality to be applied in plasmonic integration circuits. The switching threshold power, and the transmission spectra for the plasmonic switch are achieved. The propagation of electromagnetic waves in the time domain is simulated with the Finite difference time domain (FDTD) (Taflove and Hagness 2005).

\section{Theory And Structure}


In this study, MIM structures, cavities, and nonlinear characteristics of gold are employed to implement a plasmonic switch. Using high Kerr coefficient material, cavities, and inserting a piece of gold inside the cavities, result in an all-optical switch with a low power consumption and fast speed. For the first time, in addition to the material with a high nonlinear Kerr coefficient, the inherently nonlinear characteristics of the gold are the other factor used to implement a plasmonic switch. Because the high light intensity results in significant nonlinear effects in gold that might be due to displacement of free and bonded electrons (Abajo 2008; Dadap et al. 2009; Feng et al. 2012; Gramotnev and Bozhevolnyi 2010; Lin 2011; Taher-Rahmati and Granpayeh 2014).

Figure 1, shows a schematic of the proposed switch comprising a bus waveguide side coupled with a pair of asymmetric vertical cavities. The complex dielectric constant of silver is determined by the Drude model (Nozhat and Granpayeh 2014):

$$
\varepsilon_{\mathrm{m}}=\varepsilon_{\infty}-\omega_{\mathrm{p}}^{2} /\left(\omega^{2}-\mathrm{j} \gamma \omega\right)
$$

, where $\varepsilon_{\infty}, \omega_{p}, Y_{p}$, and $\omega$ are the relative permittivity at the infinite frequency, the plasma frequency, the electron collision frequency, and the angular frequency of the incident light wave, respectively. In this paper, the parameters are the following: $\varepsilon_{\infty}=1.95, \omega_{p}=1.37 \times 10^{16}(\mathrm{rad} / \mathrm{s})$, and $\mathrm{y}=20 \times 10^{12} \mathrm{rad} / \mathrm{s}$, which taken from (Nozhat and Granpayeh 2014).

The straight waveguide and the cavities are filled with air and the nonlinear $\mathrm{Au} / \mathrm{SiO}_{2}$, respectively. The dielectric in the straight waveguides is air with a refractive index $n_{0}=1$. The dielectric in the cavities is $\mathrm{Au} / \mathrm{SiO}_{2}$ with high Kerr nonlinearity, which has the refractive index of $\mathrm{n}=1.47$ and Kerr non-linear coefficient of $\mathrm{n}_{2}=2.07 \times 10^{-9} \mathrm{~cm}^{2} / \mathrm{W}$ (Liao et al. 1998).

The width of the waveguide and the width of the two asymmetric vertical cavities, $w$, are assumed to be $150 \mathrm{~nm}$. Also, the distance between the cavities, $d$, is assumed to be $150 \mathrm{~nm}$. A $100 \times 100 \mathrm{~nm}$ square of gold is inserted inside the cavities.

When light with TM polarization is injected into the MIM structure, it couples to the waveguide and SPP waves propagate along the common metal surfaces. If the wavelength of the applied light is the same as the resonant cavity wavelength, the light does not pass. This wavelength is sensitive to the dielectric constant that can be changed due to the nonlinear Kerr effect of the material. Therefore, by increasing the intensity of the input light, the wavelength changes and switching is performed. Table 1, presents the physical and geometric parameters of the proposed switch.

Table 1

Physical and geometric parameters of the switch 


\begin{tabular}{|llll|}
\hline Symbol & Parameter & Material & Quantity \\
\hline$n_{\mathrm{b}}$ & Background refractive index & $\mathrm{Ag}$ & Drude model (Liao et al. 1998) \\
\hline$n_{2}$ & Dielectric Kerr non-linear coefficient & $\mathrm{Au} / \mathrm{SiO}_{2}$ & $2.07 \mathrm{~cm}^{2} / \mathrm{GW}$ (Liao et al. 1998) \\
\hline$n_{0}$ & Dielectric linear coefficient & $\mathrm{Au} / \mathrm{SiO}_{2}$ & 1.47 (Liao et al. 1998) \\
\hline $\mathrm{w}$ & waveguide width & $\mathrm{Air}$ & $150 \mathrm{~nm}$ \\
\hline $\mathrm{d}$ & cavities distance & $\mathrm{Ag}$ & $150 \mathrm{~nm}$ \\
\hline
\end{tabular}

\section{Design And Simulation}

The asymmetric nonlinear cavity pair of the nanostructure shown in Figure 1, has a strong resonant wavelength. Figure 2, shows the transmission spectrum of the structure for low and high input intensities. Considering Figure 2, at the low intensity $\left(0.01 \mathrm{~mW} / \mu \mathrm{m}^{2}\right)$, a sharp dip occurs at the wavelength of 860 $\mathrm{nm}$. If the input light intensity is increased to $0.1 \mathrm{~mW} / \mu \mathrm{m}^{2}$, the dielectric constant of the nonlinear material also increases, and the dip is red shifted to $880 \mathrm{~nm}$.

Figure 3 , shows the dependency of signal transmission on the intensity of the input light. If the intensity of the input light changes, the dielectric constant changes and causes a difference in the transmission spectrum of the signal. Therefore, a mechanism is provided to the dual behavior of light at the output regarding the input light intensity. It should be noted that the constant dielectric change is due to the field intensity in the cavities. When the input light intensity is increased to $0.1 \mathrm{~mW} / \mu \mathrm{m}^{2}$, the signal transmission to the output increases suddenly to about 0.8 . The threshold power of the signal is about $0.07 \mathrm{~mW} / \mu \mathrm{m}^{2}$, therefore, only higher light intensity than the threshold power is required to realize the switching operation.

To demonstrate the performance of the incident light signal under on/off conditions, the magnetic field distribution of the structure for low and high light intensities is shown in Figure 4. As shown, when the input light intensity is about $0.01 \mathrm{~mW} / \mu \mathrm{m}^{2}$, the signal is reflected, and when the input light intensity increases to $0.1 \mathrm{~mW} / \mu \mathrm{m}^{2}$, it can pass the straight waveguide. The results are in good agreement with the signal transmission spectrum response under on/off conditions.

In addition to the nonlinear material with a high Kerr effect, the low light intensity required for switching is due to employing gold nanostructures inside the cavities. To ensure the validity of this issue and studying the effect of these square gold nanostructures, the proposed switch structure is studied and simulated in the absence of the gold nanostructures inside the cavities. Considering the transmission spectrum of the structure in Figure 5 for low input intensity of $0.01 \mathrm{~mW} / \mu \mathrm{m}^{2}$, a sharp dip in the transmission spectrum for through port at the resonant wavelengths of 818 and $698 \mathrm{~nm}$. The resonant is observed at a wavelength of $740 \mathrm{~nm}$. The structure is scanned at this wavelength for various light intensities and dependency of the signal transmission to the input light intensity is shown in Figure 6 . When the light intensity is 
increased to $0.2 \mathrm{~mW} / \mu \mathrm{m}^{2}$, the transmission increases to 0.62 . The threshold power of the signal is $0.15 \mathrm{~mW} / \mu \mathrm{m}^{2}$. Considering the results, using square gold nanostructures reduces the input light intensity from $0.15 \mathrm{~mW} / \mu \mathrm{m}^{2}$ to $0.07 \mathrm{~mW} / \mu \mathrm{m}^{2}$.

\section{Conclusion}

In this study, MIM structures, cavities, and intrinsic nonlinear properties of the gold are used to implement an all-optical plasmonic switch. The proposed switch is comprised of a bus waveguide side coupled with a pair of asymmetric vertical cavities. The dielectric in the waveguide and the two cavities are filled with air and Kerr nonlinear material, respectively. A $100 \times 100 \mathrm{~nm}$ square of gold is also inserted inside the cavities. The transmission spectrum showed that for low input intensities, a sharp dip occurs at the wavelength of $860 \mathrm{~nm}$. When the input light intensity is increased to $0.1 \mathrm{~mW} / \mu \mathrm{m}^{2}$, the dip of the transmission spectrum changes and the transmission power increases to 0.8 . The threshold power of the structure with and without using the gold nanostructure is $0.15 \mathrm{~mW} / \mu \mathrm{m}^{2}$ and $0.07 \mathrm{~mW} / \mu \mathrm{m}^{2}$, respectively showing a reduction of $50 \%$.

\section{Declarations}

\section{Funding}

'Not applicable'

Conflicts of interest/Competing interests

'Not applicable'

\section{Availability of data and material}

'Not applicable'

Code availability

'Not applicable'

\section{Authors' contributions}

'Not applicable'

\section{References}

Abajo, F. J. G.: Nonlocal effects in the plasmons of strongly interacting nanoparticles, dimers, and waveguides. J. Phys. Chem. C. 112, 17983-17987, (2008) 
Bashiri, S., Fasihi, K.: A $2 \times 1$ all-optical multiplexer using Kerr nonlinear nano-plasmonic switch. Opt Quant Electron 51, 374 (2019). https://doi.org/10.1007/s11082-019-2080-9

Dadap, J. I., Aguiar, H. B., Roke, S.: Nonlinear light scattering from clusters and single particles. J. Chem. Phys. 130, 214710-1-214710-7, (2009)

Emadi, R., Safian, R., Nezhad, A.Z., Emadi, R.: Analysis and Design of Graphene-Based Surface Plasmon Waveguide Switch at Long-Wavelength Infrared Frequencies, IEEE J. Sel. Top. Quant., 23 1-9, (2017)

Fasihi, K.: High-Contrast All-Optical Controllable Switching and Routing in Nonlinear Photonic Crystals, J. Lightwave Technol. 32, 18, 3126-3131, (2014)

Feng, S., Zhang, X., Li, J., Klar, P. J.: Coupling between the plasmonic and photonic resonance modes in wave-guided metallic photonic crystals, J. Nanophotonics. 6, 1, 063513-1-063513-10, (2012)

Ghadrdan M., Mansouri-Birjandi, M. A.: Concurrent implementation of all-optical half-adder and AND \& XOR logic gates based on nonlinear photonic crystal, Opt. Quant. Electron. 45, 10, 1027-1036, (2013)

Ghadrdan M., Mansouri-Birjandi, M. A.: Design and implementation of optical switches based on nonlinear plasmonic ring resonators: circular, square and octagon, photonic. Nanostruct. 29, 15-21, (2018)

Ghadrdan, M., Mansouri-Birjandi, M. A.: Implementation of all-optical switch based on nonlinear photonic crystal ring resonator with embedding metallic nanowires in the ring resonators, Opt. Quant. Electron., 48, 5, 299, (2016)

Ghadrdan, M., Mansouri-Birjandi, M. A.: Low-threshold photonic crystal all-optical switch using plasmonic nanowires placed in nonlinear resonator structure, J. Nanophotonics, 11, 3, 036017, (2017)

Gramotnev D. K., Bozhevolnyi, S. I.: Plasmonics beyond the diffraction limit. Nat. Photon. 4, 2, 83-91, (2010). https://doi.org/10.1038/nphoton.2009.282

He, Z., Li, H., Zhan, S., Li, B., Chen, Z., Xu, H.:Tunable Multi-switching in Plasmonic Waveguide with Kerr Nonlinear Resonator, Sci. Rep. 5, 15837, (2015)

Hocini, A., Ben salah, H., Khedrouche, D. et al. A high-sensitive sensor and band-stop filter based on intersected double ring resonators in metal-insulator-metal structure. Opt Quant Electron 52, 336 (2020). https://doi.org/10.1007/s11082-020-02446-x

Janjan, B., Fathi, D., Miri, M., Ghaffari-Miab, M.: Ultra-wideband high-speed Mach-Zehnder switch based on hybrid plasmonic waveguides, Appl. Opt. 56, 1717-1723, (2017)

Kik, P.G., Brongersma, M.L.: Surface Plasmon Nanophotonics, in: M.L. Brongersma, P.G. Kik (Eds.) Surface Plasmon Nanophotonics. Springer Netherlands, Dordrecht, 1-9. (2007) 
Kwon, S.-H., No, Y.-S., Park, H.-G.: Design of plasmonic cavities, Nano Convergence, 1, 8, (2014)

Liao, H. B., Xiao, R. ,F., Fu, J. S., Wang, H., Wong, K. S., Wong, G. K. L.: Origin of third-order optical nonlinearity in $\mathrm{Au}: \mathrm{SiO}_{2}$ composite films on femtosecond and picosecond time scales. Opt. Lett., 23, 388390, (1998)

Lin, J.-T.: Nonlinear optical theory and figure of merit of surface plasmon resonance of gold nanorods. J. Nanophotonics. 5, 1, 051506-051506-8, (2011)

Maier, S. A.: palsmonics, fundamentals and applications. springer, (2007)

Mansouri-Birjandi, M. A., Tavousi, A., Ghadrdan, M.: Full-optical tunable add/drop filter based on nonlinear photonic crystal ring resonators, photonic. Nanostruct. 21, 44-51, (2016)

Monfared, S.A., Seifouri, M., Hamidi, S.M. et al.: Two-dimensional graphene-plasmonic crystals for alloptical switch applications. Opt Quant Electron 52, 497 (2020). https://doi.org/10.1007/s11082-02002618-9

Negahdari, R., Rafiee, E. \& Emami, F.: Realization of all-optical plasmonic MIM split square ring resonator switch. Opt Quant Electron 51, 235 (2019). https://doi.org/10.1007/s11082-019-1924-7

Nozhat, N., Granpayeh, N.: All-optical nonlinear plasmonic ring resonator switches, Journal of Modern Optics. 61, 1690-1695, (2014)

Nozhat, N., Granpayeh, N.: Switching Power Reduction of the Plasmonic Directional Coupler by XPM Nonlinear Effect. IEEE Photonic. Tech. L. 24, 1154-1156, (2012)

Nurmohammadi, T., Abbasian, K., Yadipour, R.: Ultra-fast all-optical plasmonic switching in near infra-red spectrum using a Kerr nonlinear ring resonator. Opt. Commun., 410, 142-147, (2018).

https://doi.org/10.1016/j.optcom.2017.09.082

Paul, S., Ray, M.: Plasmonic switching and bistability at telecom wavelength using the subwavelength nonlinear cavity coupled to a dielectric waveguide: A theoretical approach, J. Appl. Phys. 120, 203102, (2016)

Petráček, J.: Nonlinear directional coupling between plasmonic slot waveguides. Appl. Phys. B. 112, 593598, (2013)

Sasikala, V., Chitra, K.: All optical switching and associated technologies: a review. J Opt., 47, 307-317, (2018). https://doi.org/10.1007/s12596-018-0452-3

Shahamat, Y., Vahedi, M.: Designing ultra-compact high efficiency electro-optical plasmonic switches by using of nanocavity reflectors, Opt. Commun., 410, 25-29, (2018).

https://doi.org/10.1016/j.optcom.2017.09.083 
Taflove A., Hagness, S. C.: Computational Electrodynamics: The Finite-difference Time-domain Method (Artech House antennas and propagation library). Artech House, (2005)

Taher Rahmati, A., Granpayeh, N.: Low Power Nonlinear Active Devices Based on Intrinsic Metal Nonlinearities, J. Lightwave Technol. 32, 21, (2014)

Wu, T., Liu, Y., Yu, Z., Peng, Y., Shu, C., Ye, H.: The sensing characteristics of plasmonic waveguide with a ring resonator. Opt. Express, 22, 7669-7677, (2014)

Wu, Y.D., Liu, C.L., Jhan, G.Y.: All-optical switch based on the local nonlinear plasmonic Mach-Zehnder interferometer waveguides, International Conference on Numerical Simulation of Optoelectronic Devices (NUSOD), 51-52., (2015)

Zhang X., Yang, J.: Ultrafast Plasmonic Optical Switching Structures and Devices. Front. Phys. 7, no.190, (2019). doi: 10.3389/fphy.2019.00190

\section{Figures}

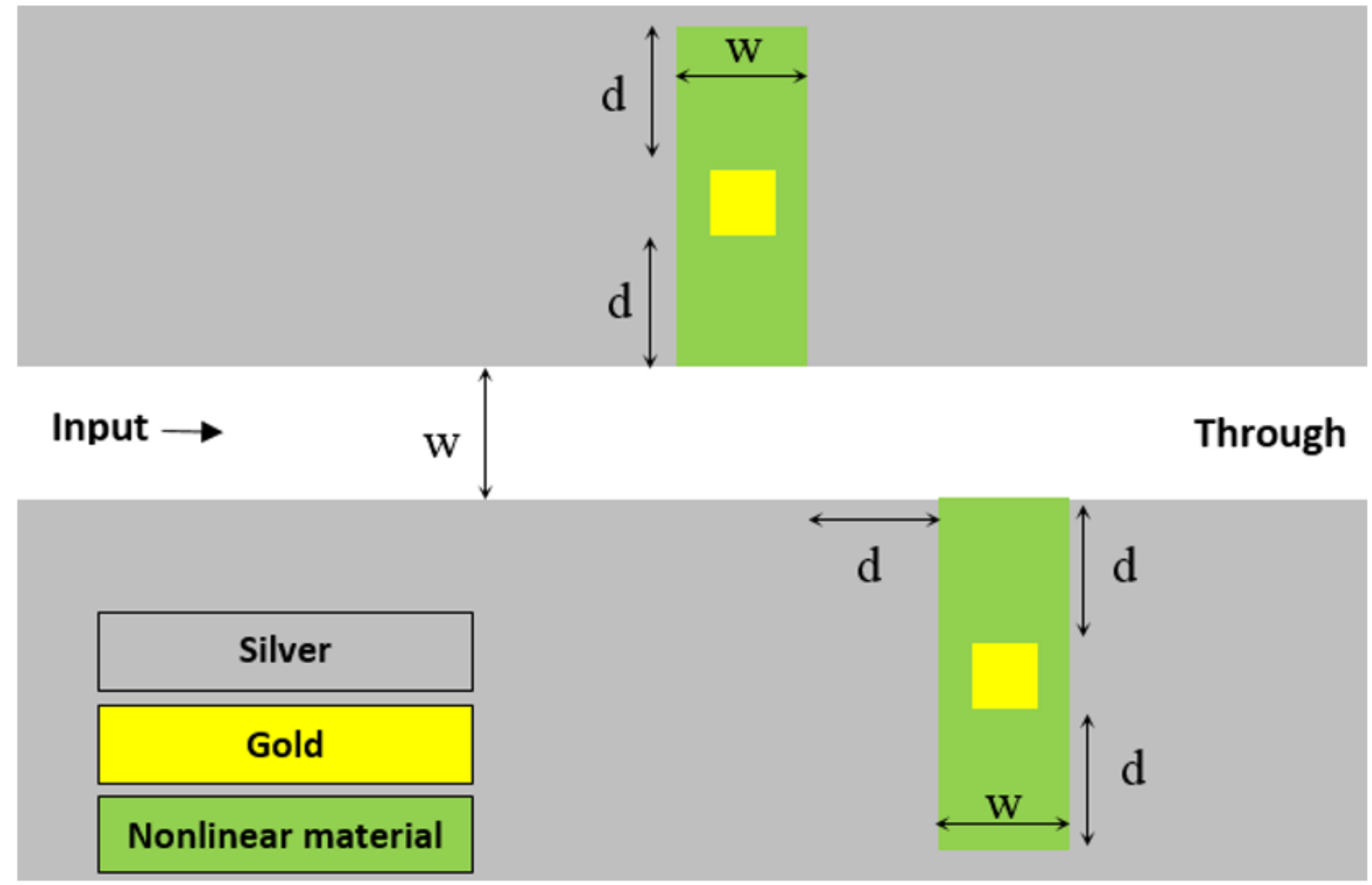

Figure 1 
The schematic of the proposed plasmonic switch

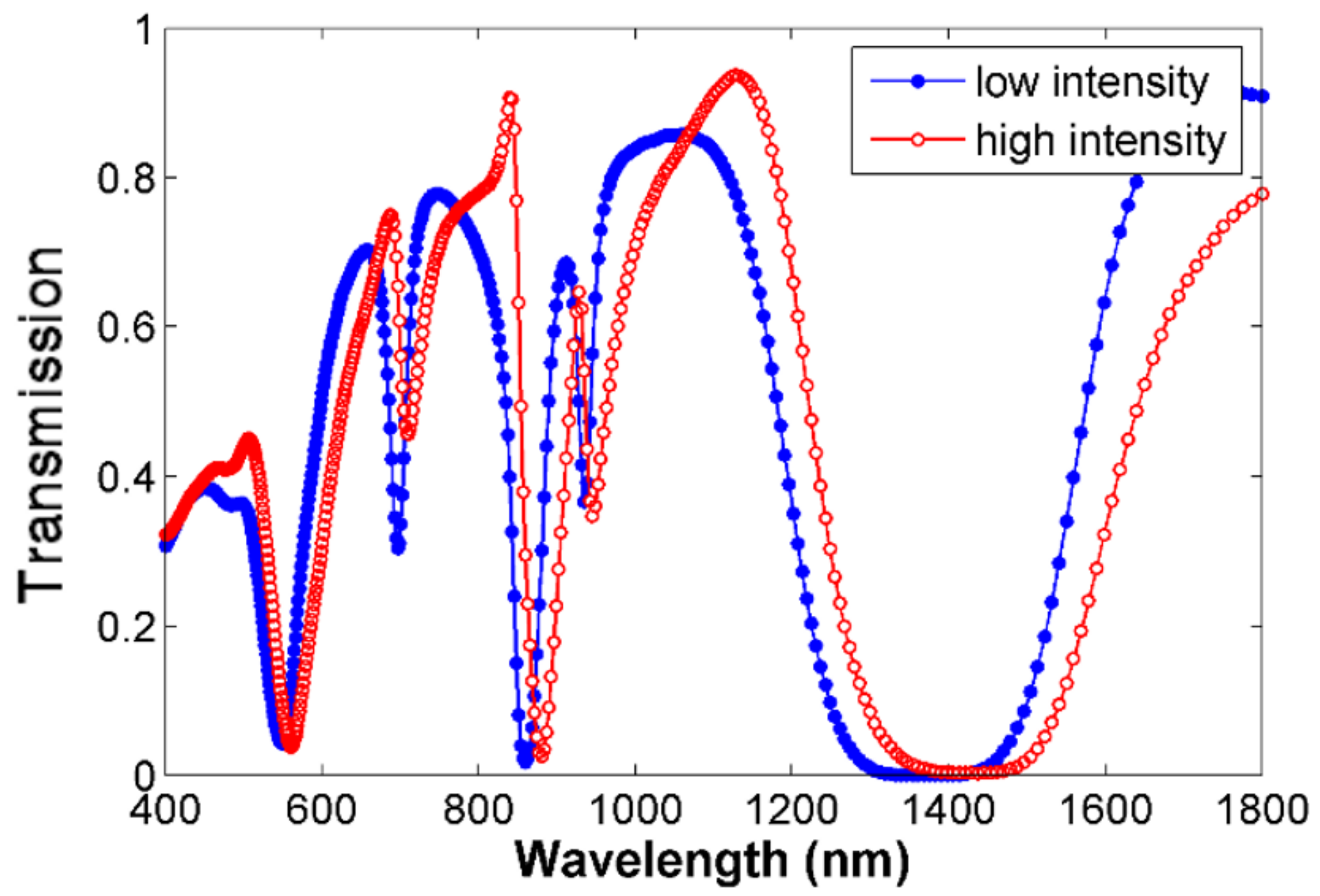

Figure 2

The transmission spectrum of the proposed plasmonic switch for low and high input intensities 


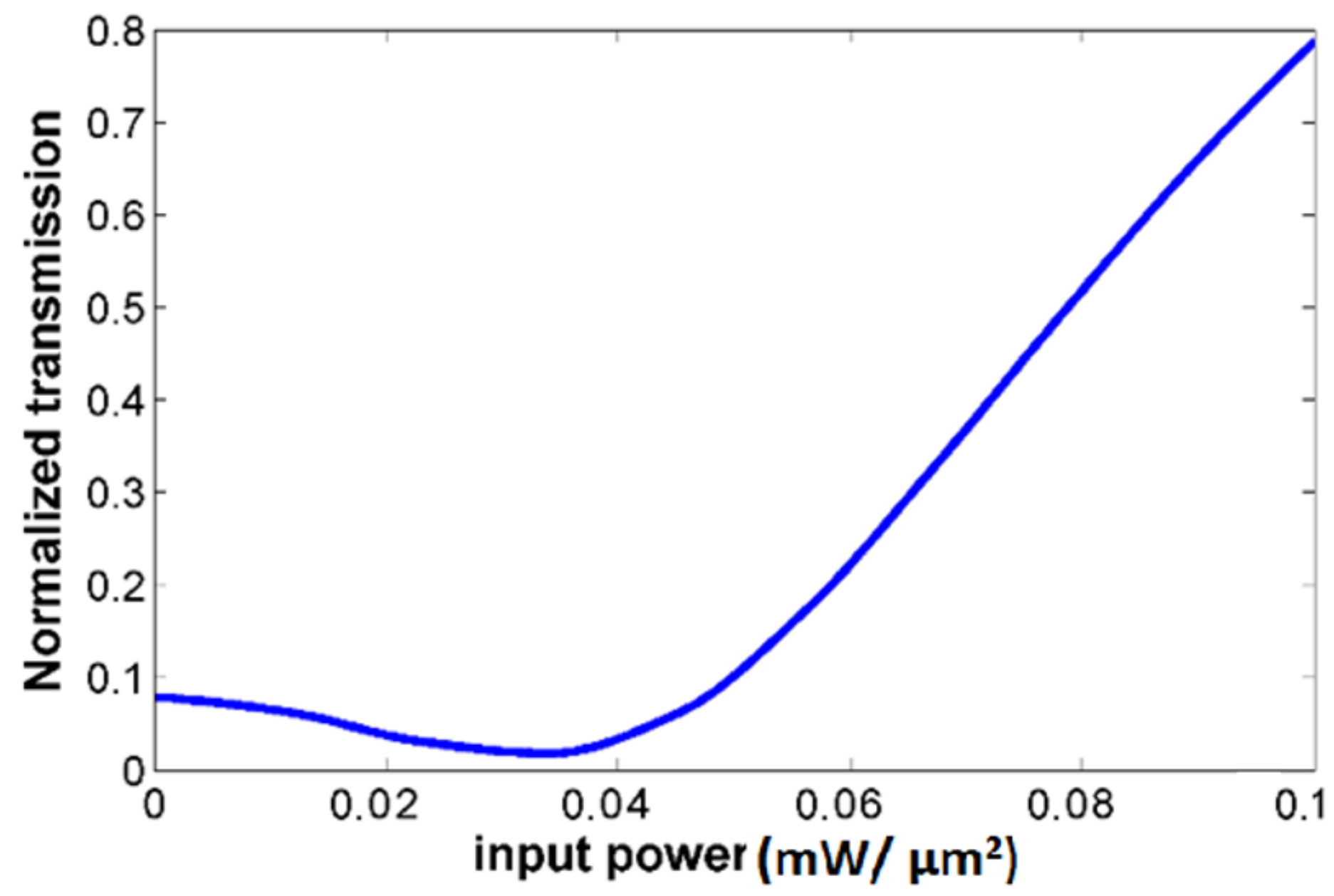

Figure 3

The dependency of signal transmission to the intensity of the input light with gold nanostructures 

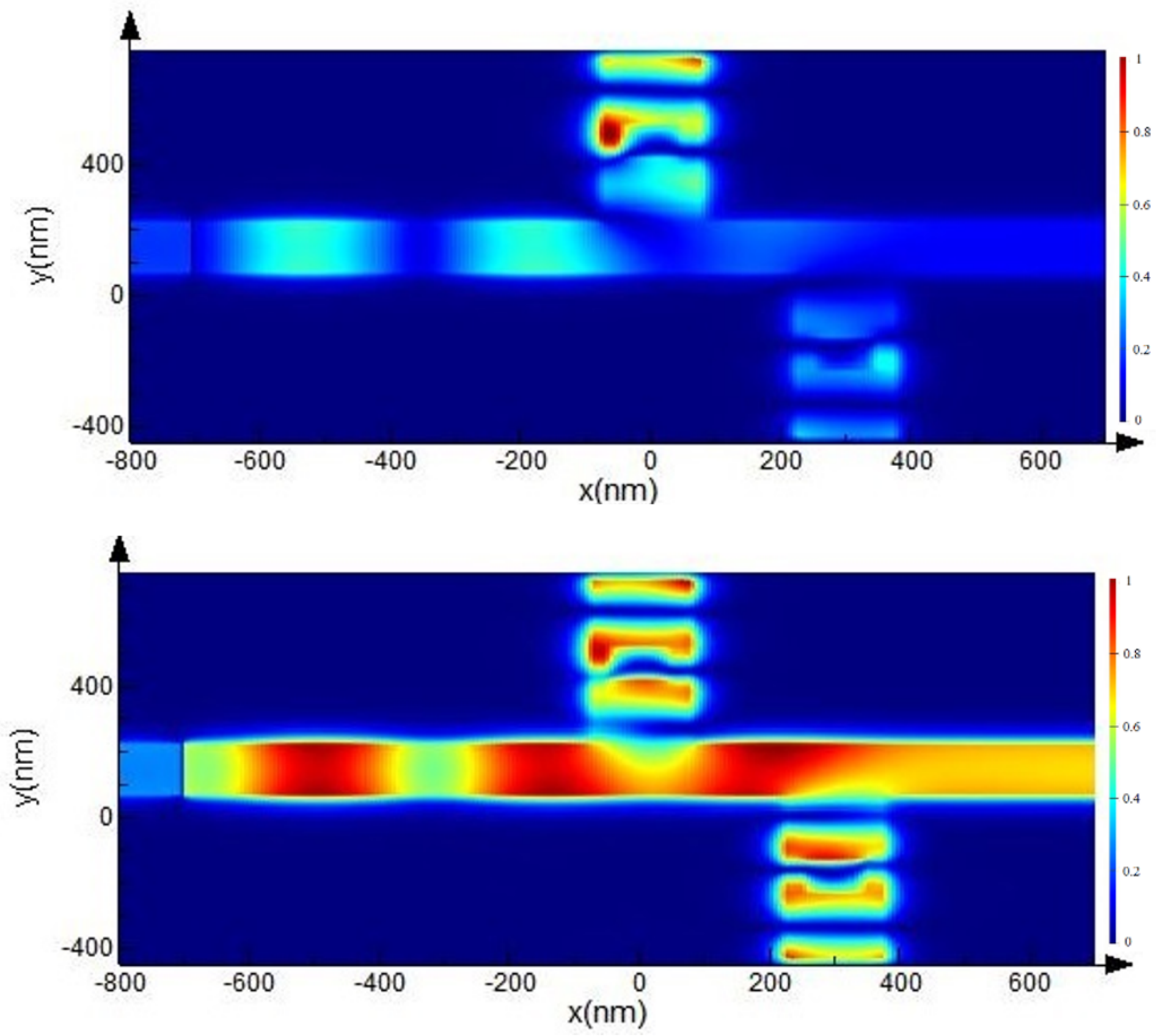

Figure 4

The magnetic field distribution of the structure for low and high light intensities 


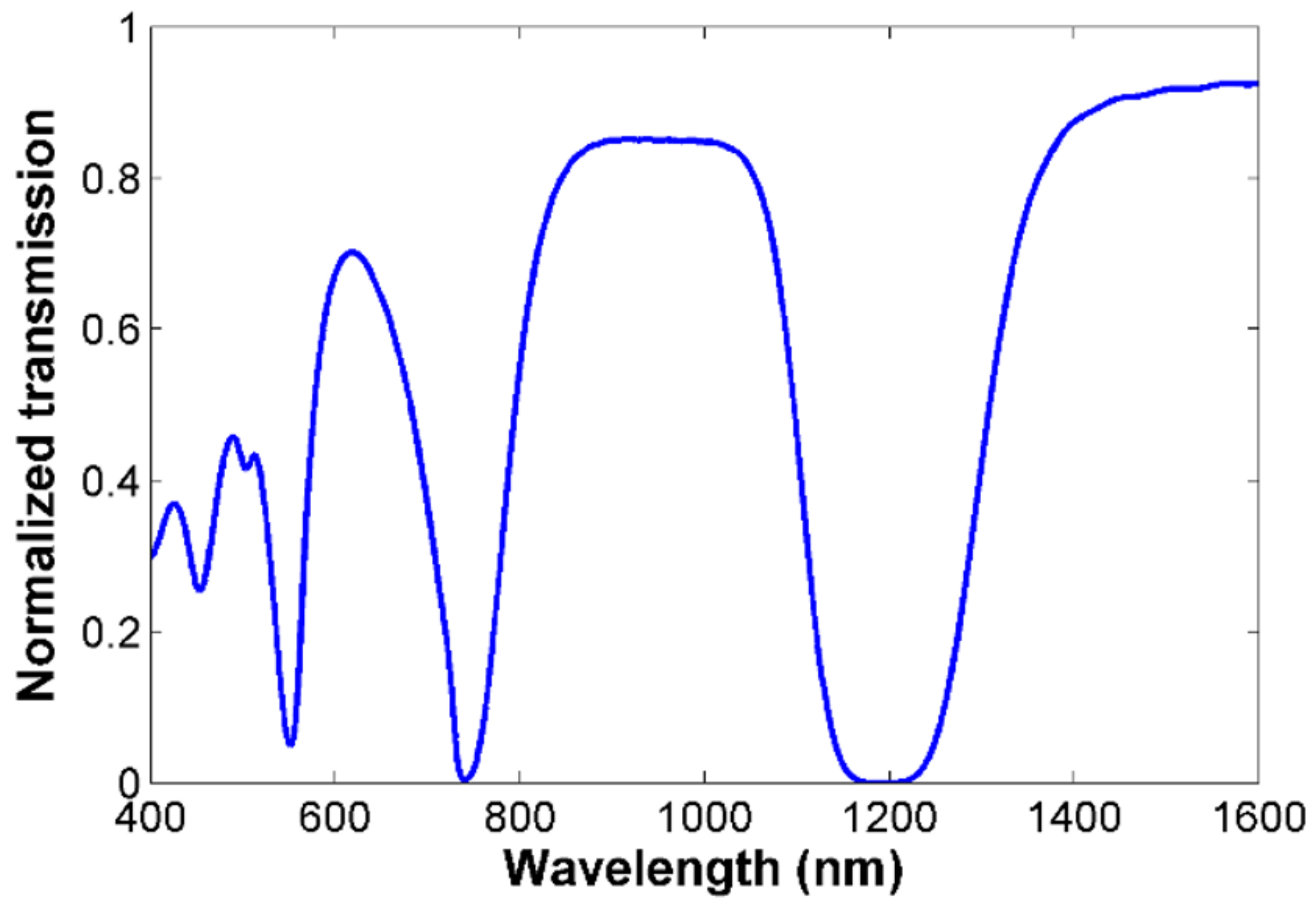

Figure 5

The transmission spectrum of the proposed plasmonic switch without gold nanostructures 


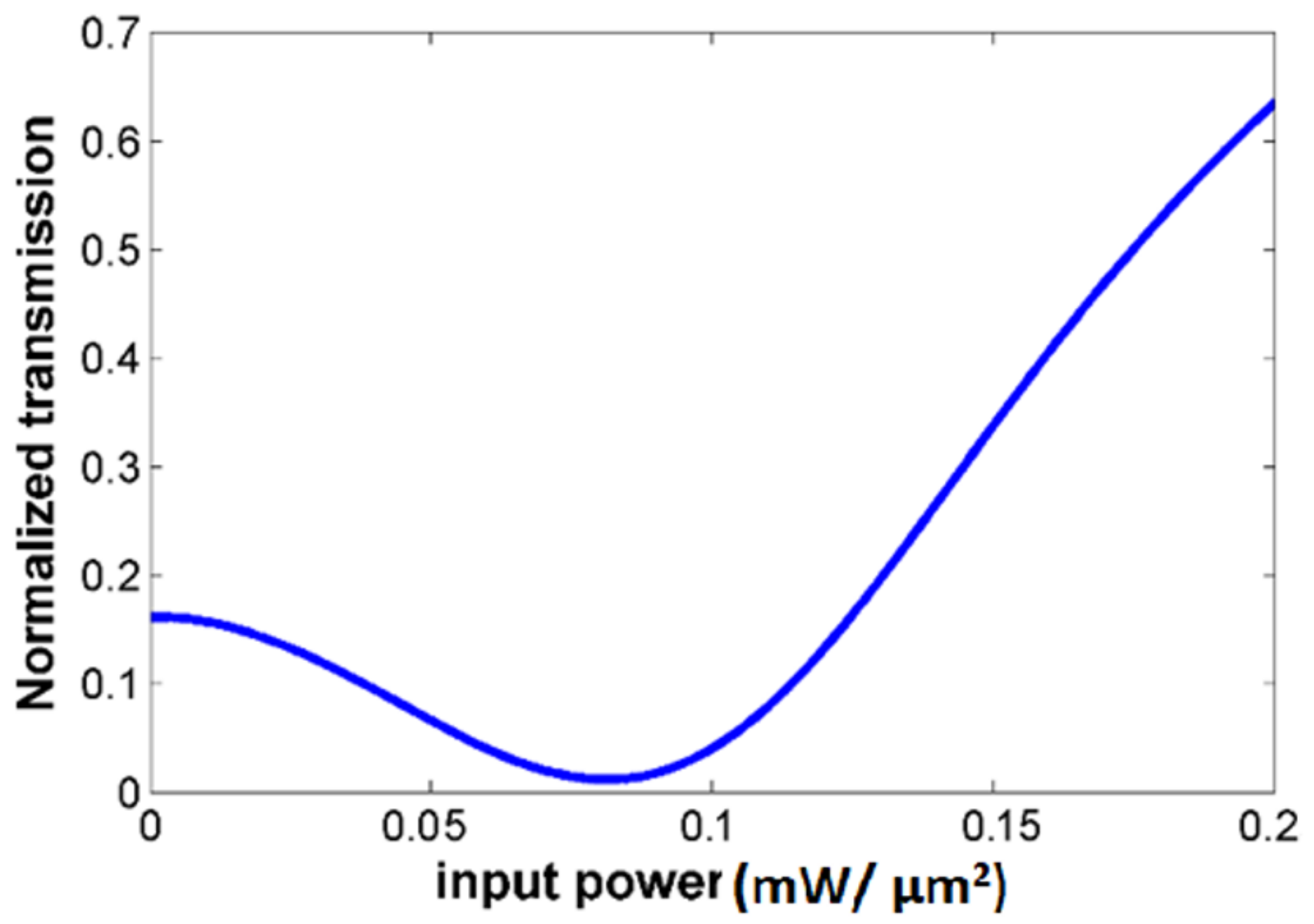

Figure 6

The dependency of the signal transmission to the input light intensity without gold nanostructures 